\title{
A New Analytical Solution for the Distance Modulus in Flat Cosmology
}

\author{
Lorenzo Zaninetti \\ Physics Department, via P. Giuria 1, Turin, Italy \\ Email: zaninetti@ph.unito.it
}

How to cite this paper: Zaninetti, L. (2019) A New Analytical Solution for the Distance Modulus in Flat Cosmology. International Journal of Astronomy and Astrophysics, 9, 51-62. https://doi.org/10.4236/ijaa.2019.91005

Received: January 11, 2019

Accepted: March 11, 2019

Published: March 14, 2019

Copyright $\odot 2019$ by author(s) and Scientific Research Publishing Inc. This work is licensed under the Creative Commons Attribution International License (CC BY 4.0).

http://creativecommons.org/licenses/by/4.0/

\begin{abstract}
A new analytical solution for the luminosity distance in flat $\Lambda \mathrm{CDM}$ cosmology is derived in terms of elliptical integrals of first kind with real argument. The consequent derivation of the distance modulus allows evaluating the Hubble constant, $H_{0}=69.77 \pm 0.33, \Omega_{\mathrm{M}}=0.295 \pm 0.008$ and the cosmological constant, $\Lambda=(1.194 \pm 0.017) \times 10^{-52} \frac{1}{\mathrm{~m}^{2}}$.
\end{abstract}

\section{Keywords}

Galaxy Groups, Clusters, and Superclusters, Large Scale Structure of the Universe, Cosmology

\section{Introduction}

The release of two catalogs for the distance modulus of Supernova (SN) of type Ia, namely, the Union 2.1 compilation, see [1], and the joint light-curve analysis (JLA), see [2], allows matching the observed distance modulus with the theoretical distance modulus of various cosmologies. In this fitting procedure, the cosmological parameters are derived in a scientific and reproducible way.

We now focus our attention on the flat Friedmann-Lemaitre-Robertson-Walker (flat-FLRW) cosmology. A first fitting formula has been derived by [3] and an approximate solution in terms of Padé approximant has been introduced by [4]. The presence of the elliptical integrals of the first kind in the integral for the luminosity distance in flat-FLRW cosmology has been noted by [5] [6] [7]. As a practical example the luminosity distance can be expanded into a series of orthonormal functions and the two cosmological parameters turn out to be $H_{0}=70.43 \pm 0.33$ and $\Omega_{\mathrm{M}}=0.297 \pm 0.002$, see [8]. This paper first introduces in Section 2 a framework useful to build a new solution for the luminosity dis- 
tance in flat-FLRW cosmology, which will be derived in Section 3.

\section{Preliminaries}

This section reviews the adopted statistical framework, the $\Lambda \mathrm{CDM}$ cosmology, and an existing solution for the luminosity distance in flat-FLRW cosmology.

\subsection{The Adopted Statistics}

In the case of the distance modulus, the merit function $\chi^{2}$ is

$$
\chi^{2}=\sum_{i=1}^{N}\left[\frac{(m-M)_{i}-(m-M)\left(z_{i}\right)_{t h}}{\sigma_{i}}\right]^{2},
$$

where $N$ is the number of SNs, $(m-M)_{i}$ is the observed distance modulus evaluated at redshift $z_{i}, \sigma_{i}$ is the error in the observed distance modulus evaluated at $z_{i}$, and $(m-M)\left(z_{i}\right)_{t h}$ is the theoretical distance modulus evaluated at $z_{i}$, see formula (15.5.5) in [9]. The reduced merit function $\chi_{\text {red }}^{2}$ is

$$
\chi_{\text {red }}^{2}=\chi^{2} / N F \text {, }
$$

where $N F=N-k$ is the number of degrees of freedom, $N$ is the number of SNs, and $k$ is the number of parameters. Another useful statistical parameter is the associated $Q$-value, which has to be understood as the maximum probability of obtaining a better fitting, see formula (15.2.12) in [9]:

$$
Q=1-\operatorname{GAMMQ}\left(\frac{N-k}{2}, \frac{\chi^{2}}{2}\right),
$$

where GAMMQ is a subroutine for the incomplete gamma function.

The goodness of the approximation in evaluating a physical variable $p$ is evaluated by the percentage error $\delta$

$$
\delta=\frac{\left|p-p_{\text {approx }}\right|}{p} \times 100,
$$

where $p_{\text {approx }}$ is an approximation of $p$.

\subsection{The Standard Cosmology}

We follow [10], where the Hubble distance $D_{\mathrm{H}}$ is defined as

$$
D_{\mathrm{H}} \equiv \frac{c}{H_{0}} .
$$

The first parameter is $\Omega_{\mathrm{M}}$

$$
\Omega_{\mathrm{M}}=\frac{8 \pi G \rho_{0}}{3 H_{0}^{2}},
$$

where $G$ is the Newtonian gravitational constant and $\rho_{0}$ is the mass density at the present time. The second parameter is $\Omega_{\Lambda}$

$$
\Omega_{\Lambda} \equiv \frac{\Lambda c^{2}}{3 H_{0}^{2}},
$$


where $\Lambda$ is the cosmological constant, see [11]. These two parameters are connected with the curvature $\Omega_{K}$ by

$$
\Omega_{\mathrm{M}}+\Omega_{\Lambda}+\Omega_{K}=1 \text {. }
$$

The comoving distance, $D_{\mathrm{C}}$, is

$$
D_{\mathrm{C}}=D_{\mathrm{H}} \int_{0}^{z} \frac{\mathrm{d} z^{\prime}}{E\left(z^{\prime}\right)}
$$

where $E(z)$ is the "Hubble function"

$$
E(z)=\sqrt{\Omega_{\mathrm{M}}(1+z)^{3}+\Omega_{K}(1+z)^{2}+\Omega_{\Lambda}} .
$$

The above integral does not have an analytical solution but a solution in terms of Padé approximant has been found, see [12].

\subsection{A First Formula for a Flat-FLRW Universe}

The first model starts from Equation (2.1) in [4] for the luminosity distance, $d_{\mathrm{L}}$,

$$
d_{\mathrm{L}}\left(z ; c, H_{0}, \Omega_{\mathrm{M}}\right)=\frac{c}{H_{0}}(1+z) \int_{\frac{1}{1+z}}^{1} \frac{\mathrm{d} a}{\sqrt{\Omega_{\mathrm{M}} a+\left(1-\Omega_{\mathrm{M}}\right) a^{4}}},
$$

where $H_{0}$ is the Hubble constant expressed in $\mathrm{km} \cdot \mathrm{s}^{-1} \cdot \mathrm{Mpc}^{-1}, c$ is the speed of light expressed in $\mathrm{km} \cdot \mathrm{s}^{-1}, z$ is the redshift and $a$ is the scale-factor. The indefinite integral, $\Phi(a)$, is

$$
\Phi\left(a, \Omega_{\mathrm{M}}\right)=\int \frac{\mathrm{d} a}{\sqrt{\Omega_{\mathrm{M}} a+\left(1-\Omega_{\mathrm{M}}\right) a^{4}}} .
$$

The solution is in terms of $F$, the Legendre integral or incomplete elliptic integral of the first kind, and is given in [13].

The luminosity distance is

$$
d_{\mathrm{L}}\left(z ; c, H_{0}, \Omega_{\mathrm{M}}\right)=\mathfrak{R}\left(\frac{c}{H_{0}}(1+z)\left(\Phi(1)-\Phi\left(\frac{1}{1+z}\right)\right)\right),
$$

where $\mathfrak{R}$ means the real part. The distance modulus is

$$
(m-M)=25+5 \log _{10}\left(d_{\mathrm{L}}\left(z ; c, H_{0}, \Omega_{\mathrm{M}}\right)\right) .
$$

\section{A New Formula for a Flat-FLRW Universe}

The second model for the flat cosmology starts from Equation (1) for the luminosity distance in [14]

$$
d_{\mathrm{L}}\left(z ; c, H_{0}, \Omega_{\mathrm{M}}\right)=\frac{c(1+z)}{H_{0}} \int_{0}^{z} \frac{1}{\sqrt{\Omega_{\mathrm{M}}(1+t)^{3}+1-\Omega_{\mathrm{M}}}} \mathrm{d} t .
$$

The above formula can be obtained from formula (9) for the comoving distance inserting $\Omega_{K}=0$ and the variable of integration, $t$, denotes the redshift.

A first change in the parameter $\Omega_{\mathrm{M}}$ introduces

$$
s=\sqrt[3]{\frac{1-\Omega_{\mathrm{M}}}{\Omega_{\mathrm{M}}}}
$$


and the luminosity distance becomes

$$
d_{\mathrm{L}}\left(z ; c, H_{0}, s\right)=\frac{1}{H_{0}} c(1+z) \int_{0}^{z} \frac{1}{\sqrt{\frac{(1+t)^{3}}{s^{3}+1}+1-\left(s^{3}+1\right)^{-1}}} \mathrm{~d} .
$$

The following change of variable, $t=\frac{s-u}{u}$, is performed for the luminosity distance which becomes

$$
d_{\mathrm{L}}\left(z ; c, H_{0}, s\right)=-\frac{c}{H_{0} s^{2}}(1+z)\left(s^{3}+1\right) \int_{s}^{\frac{s}{1+z}} \frac{u}{u^{3}+1} \sqrt{\frac{s^{3}\left(u^{3}+1\right)}{u^{3}\left(s^{3}+1\right)}} \mathrm{d} u .
$$

Up to now we have followed [14] which continues introducing a new function $T(x)$; conversely we work directly on the resulting integral for the luminosity distance: which is

$$
\begin{aligned}
& d_{\mathrm{L}}\left(z ; c, H_{0}, s\right) \\
& =-1 / 3 \frac{c(1+z) 3^{3 / 4} \sqrt{s^{3}+1}}{\sqrt{s} H_{0}} \times\left(F\left(2 \frac{\sqrt{s(s+1+z)} \sqrt[4]{3}}{s \sqrt{3}+s+z+1}, 1 / 4 \sqrt{2} \sqrt{3}+1 / 4 \sqrt{2}\right)\right. \\
& \left.-F\left(2 \frac{\sqrt[4]{3} \sqrt{s(s+1)}}{s+1+s \sqrt{3}}, 1 / 4 \sqrt{2} \sqrt{3}+1 / 4 \sqrt{2}\right)\right)
\end{aligned}
$$

where $s$ is given by Equation (16) and $F(\phi, k)$ is Legendre's incomplete elliptic integral of the first kind,

$$
F(\phi, k)=\int_{0}^{\sin \phi} \frac{\mathrm{d} t}{\sqrt{1-t^{2}} \sqrt{1-k^{2} t^{2}}},
$$

see [15]. The distance modulus is

$$
(m-M)=25+5 \log _{10}\left(d_{\mathrm{L}}\left(z ; c, H_{0}, s\right)\right),
$$

and therefore

$$
(m-M)=25+5 \frac{1}{\ln (10)} \ln \left(-\frac{1}{3} \frac{c(1+z) 3^{3 / 4}\left(F_{1}-F_{2}\right) \sqrt{s^{3}+1}}{\sqrt{s} H_{0}}\right),
$$

where

$$
F_{1}=F\left(2 \frac{\sqrt{s(s+1+z)} \sqrt[4]{3}}{s \sqrt{3}+s+z+1}, 1 / 4 \sqrt{2} \sqrt{3}+1 / 4 \sqrt{2}\right)
$$

and

$$
F_{2}=F\left(2 \frac{\sqrt[4]{3} \sqrt{s(s+1)}}{s+1+s \sqrt{3}}, 1 / 4 \sqrt{2} \sqrt{3}+1 / 4 \sqrt{2}\right)
$$

with $s$ as defined by Equation (16).

\section{Data Analysis}

In recent years, the extraction of the cosmological parameters from the distance 
modulus of SNs has become a common practice, see among others [8] [16] [17]. The best fit to the distance modulus of SNs is here obtained by implementing the Levenberg-Marquardt method (subroutine MRQMIN in [9]). This method requires the fitting function, in our case Equation (22), as well the first derivative $\frac{\partial(m-M)}{\partial H_{0}}$, which has a simple expression, and the first derivative $\frac{\partial(m-M)}{\partial \Omega_{\mathrm{M}}}$, which has a complicated expression. A simplification can be introduced by imposing a fiducial value for the Hubble constant, namely $H_{0}=70 \mathrm{~km} \cdot \mathrm{s}^{-1} \cdot \mathrm{Mpc}^{-1}$, see [2] [18]. We call this model "flat-FLRW-1", where the "1" stands for there being one parameter. Table 1 presents $H_{0}$ and $\Omega_{\mathrm{M}}$ for the Union 2.1 compilation of SNs and Figure 1 displays the best fit. The reading of this table allows to evaluate the goodness of the approximation, see (4), in the derivation of the Hubble constant in going from the supposed true value $\left(H_{0}=70 \mathrm{~km} \cdot \mathrm{s}^{-1} \cdot \mathrm{Mpc}^{-1}\right)$ to the deduced value $\left(H_{0}=69.77 \mathrm{~km} \cdot \mathrm{s}^{-1} \cdot \mathrm{Mpc}^{-1}\right)$, which is $\delta=99.67 \%$. The

Table 1. Numerical values from the Union 2.1 compilation of $\chi^{2}, \chi_{\text {red }}^{2}$ and $Q$, where $k$ stands for the number of parameters.

\begin{tabular}{|c|c|c|c|c|c|c|}
\hline Cosmology & SNs & $k$ & parameters & $\chi^{2}$ & $\chi_{\text {red }}^{2}$ & $Q$ \\
\hline flat-FLRW & 580 & 2 & $\begin{aligned} H_{0} & =69.77 \pm 0.33 ; \\
\Omega_{\mathrm{M}} & =0.295 \pm 0.008\end{aligned}$ & 562.55 & 0.9732 & 0.66 \\
\hline flat-FLRW-1 & 580 & 1 & $\begin{array}{c}H_{0}=70 ; \\
\Omega_{\mathrm{M}}=0.295 \pm 0.008\end{array}$ & 563.52 & 0.9732 & 0.669 \\
\hline$\Lambda \mathrm{CDM}$ & 580 & 3 & $\begin{array}{c}H_{0}=69.81 ; \quad \Omega_{\mathrm{M}}=0.239 ; \\
\Omega_{\Lambda}=0.651\end{array}$ & 562.61 & 0.975 & 0.658 \\
\hline
\end{tabular}

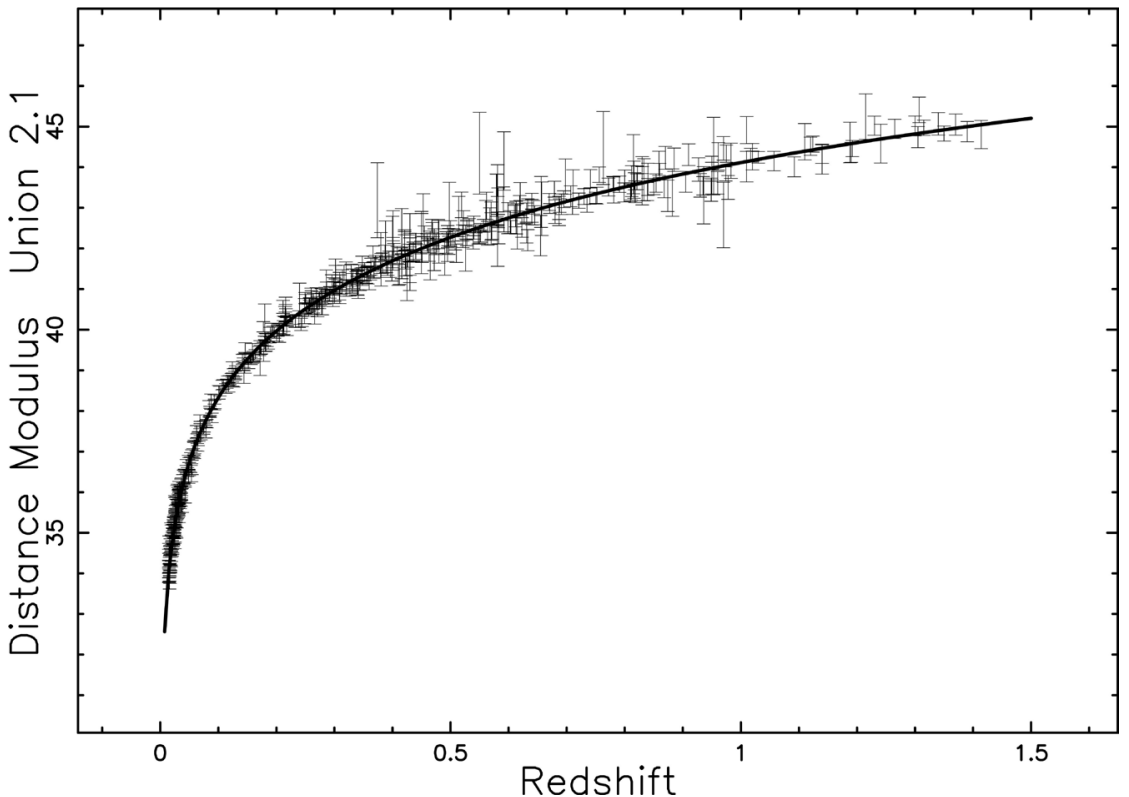

Figure 1. Hubble diagram for the Union 2.1 compilation. The solid line represents the best fit for the exact distance modulus in flat-FLRW cosmology as represented by Equation (22). Parameters as in first line of Table 1; Union 2.1 compilation. 
JLA compilation is available at the Strasbourg Astronomical Data Centre (CDS), and consists of 740 type I-a SNs for which we have the heliocentric redshift, $z$, the apparent magnitude $m_{B}^{\star}$ in the $B$ band, the error in $m_{B}^{\star}, \sigma_{m_{B}^{\star}}$, the parameter $X 1$, the error in $X 1, \sigma_{X 1}$, the parameter $C$, the error in $C, \sigma_{C}$, and $\log _{10}\left(M_{\text {stellar }}\right)$. The observed distance modulus is defined by Equation (4) in [2]:

$$
m-M=-C \beta+X 1 \alpha-M_{b}+m_{B}^{\star} .
$$

The adopted parameters are $\alpha=0.141, \beta=3.101$ and

$$
M_{b}= \begin{cases}-19.05 & \text { if } M_{\text {stellar }}<10^{10} M_{\odot} \\ -19.12 & \text { if } M_{\text {stellar }} \geq 10^{10} M_{\odot}\end{cases}
$$

see line 1 in Table 10 of [2]. The uncertainty in the observed distance modulus, $\sigma_{m-M}$, is found by implementing the error propagation equation (often called the law of errors of Gauss) when the covariant terms are neglected, see Equation (3.14) in [19],

$$
\sigma_{m-M}=\sqrt{\alpha^{2} \sigma_{X 1}^{2}+\beta^{2} \sigma_{C}^{2}+\sigma_{m_{B}^{ \pm}}^{2}} .
$$

The parameters as derived from the JLA compilation are presented in Table 2 and the fit is presented in Figure 2.

Table 2. Numerical values from the JLA compilation of $\chi^{2}, \chi_{\text {red }}^{2}$ and $Q$, where $k$ stands for the number of parameters.

\begin{tabular}{lcccccc}
\hline Cosmology & SNs & $k$ & parameters & $\chi^{2}$ & $\chi_{\text {red }}^{2}$ & $Q$ \\
\hline flat-FLRW & 740 & 2 & $\begin{array}{c}H_{0}=69.65 \pm 0.231 ; \\
\Omega_{\mathrm{M}}=0.3 \pm 0.003\end{array}$ & & & \\
& & & & & & 0.85 \\
\hline
\end{tabular}

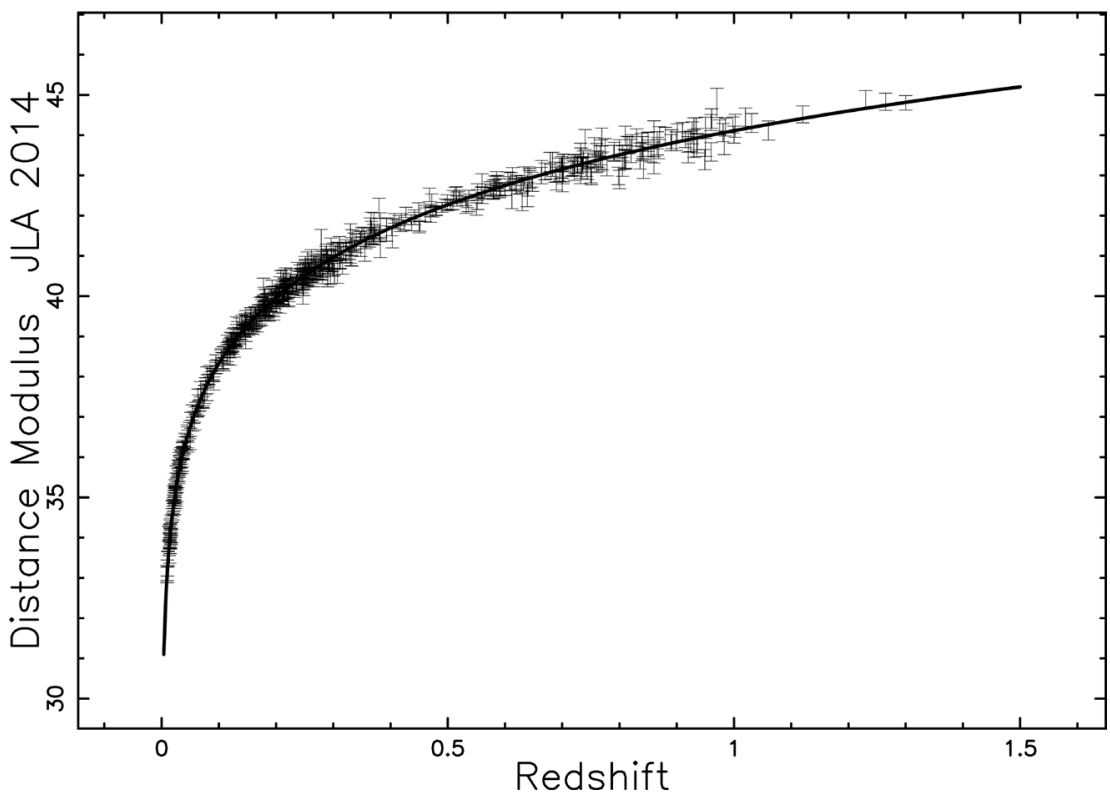

Figure 2. Hubble diagram for the JLA compilation. The solid line represents the best fit for the exact distance modulus in flat-FLRW cosmology as represented by Equation (22). Parameters as in first line of Table 2. 
As an example the luminosity distance for the Union 2.1 compilation with data as in the first line of Table 1 is

$$
\begin{aligned}
& d_{\mathrm{L}}(z)=8147.04(1.0+z) \\
& \times\left(-0.637664 F\left(2.63214 \frac{\sqrt{3.1188+1.33542 z}}{4.64846+z}, 0.965925\right)+1.75322\right) \mathrm{Mpc}
\end{aligned}
$$

when $0<z<1.5$

and the distance modulus is

$$
\begin{aligned}
& m-M=25.0+2.17147 \ln (8147.04(1.0+z) \\
& \left.\times\left(-0.63766 F\left(2.6321 \frac{\sqrt{3.1188+1.3354 z}}{4.6484+z}, 0.96592\right)+1.7532\right)\right) .
\end{aligned}
$$

when $0<z<1.5$

We now derive some approximate results without Legendre integral for the flat-FLRW case and Union 2.1 compilation with data as in Table 1, first line. A Taylor expansion of order 6 around $z=0$ of the luminosity distance as given by Equation (19) for the flat-FLRW case and Union 2.1 compilation gives

$$
\begin{aligned}
& \begin{aligned}
d_{\mathrm{L}}(z)= & 0.000423646+4296.57 z+3344.13 z^{2}-1186.94 z^{3} \\
& +979.403 z^{4}-42078.6 z^{5} \mathrm{Mpc}
\end{aligned} \\
& \text { when } 0<z<0.197 .
\end{aligned}
$$

The upper limit in redshift, 0.197 , is the value for which the percentage error, see Equation (4), is $\delta=1.16 \%$. The asymptotic expansion of the luminosity distance with respect to the variable $z$ to order 5 for the flat-FLRW case and Union 2.1 compilation gives

$$
\begin{aligned}
d_{\mathrm{L}}(z) \sim & 14283.5 z-15802 \frac{1}{\sqrt{z^{-1}}}+14283.5-7901.01 \sqrt{z^{-1}} \\
& +1975.25\left(z^{-1}\right)^{3 / 2} \mathrm{Mpc}
\end{aligned}
$$

when $1.27<z<1.5$.

At the lower limit of $z=1.27$ the percentage error is $\delta=0.54 \%$. The two above approximations at low and high redshift have a limited range of existence but does not contain the Legendre integral as solutions (28) and (29) which cover the overall range $0<z<1.5$.

A Taylor expansion of order 6 of the distance modulus as given by Equation (22) around $z=0.1$ for the flat-FLRW case and Union 2.1 compilation gives

$$
\begin{aligned}
(m-M)= & 36.0051+23.1777 z-109.604(z-0.1)^{2}+724.464(z-0.1)^{3} \\
& -5429.06(z-0.1)^{4}+43429.8(z-0.1)^{5}
\end{aligned}
$$

when $0.1<z<0.197$.

The upper limit in redshift, 0.197 , is the value at which the percentage error is $\delta=0.14 \%$. Figure 3 reports both the numerical and the Taylor expansion of distance modulus in the above range. 


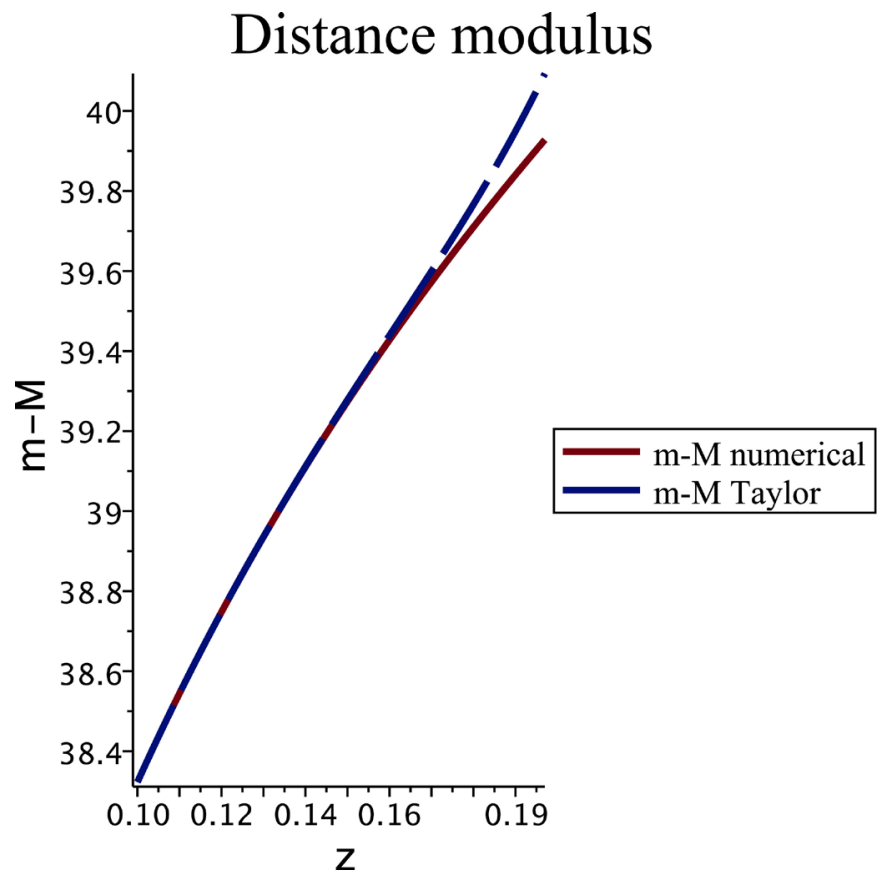

Figure 3. Distance modulus in flat-FLRW cosmology as represented by Equation (22) with parameters as in first line of Table 1 (full red line) and Taylor solution (dash-dot-dash line) (blue line).

The asymptotic expansion of the distance modulus with respect to the variable $z$ to order 5 for the flat-FLRW case and Union 2.1 compilation gives

$$
\begin{aligned}
(m-M) \sim & 45.7741+2.17147 \ln (z)-2.40231 \sqrt{z^{-1}}+0.842625 z^{-1} \\
& +0.221081\left(z^{-1}\right)^{3 / 2}-0.570086 z^{-2}-0.150471\left(z^{-1}\right)^{5 / 2} \\
& +0.357849 z^{-3}+0.491842\left(z^{-1}\right)^{7 / 2}+0.179989 z^{-4}
\end{aligned}
$$

when $1.27<z<1.5$.

The lower limit in redshift, 1.27 , is the value at which the percentage error is $\delta=0.54 \%$. The ranges of existence in $z$ for the analytical approximations here derived have the percentage error $<2 \%$, see Equation (4).

We now introduce the best minimax rational approximation, see [20] [21] [15], of degree $(2,1)$, for the distance modulus $m_{2,1}(z)$,

$$
m_{2,1}(z)=\frac{a+b z+c z^{2}}{d+e z} .
$$

In the case in which the distance modulus is represented by Equation (29) and given the interval $[0.001,1.5]$, the coefficients of the best minimax rational approximation are presented in Table 3; the maximum error for the fit is $\approx 2.2 \times 10^{-5}$. Figure 4 displays the data and the fit.

\section{Conclusions}

We have presented an analytical approximation for the luminosity distance in terms of elliptical integrals with real argument. The fit of the distance modulus 
Table 3. Maximum error and coefficients of the distance modulus for the best minimax rational approximation for the flat-FLRW case and Union 2.1 compilation. Interval of existence $[0.001,1.5]$.

\begin{tabular}{cc}
\hline Name & value \\
\hline maximum error & $2.28881836 \times 10^{-5}$ \\
a & 0.981622279 \\
b & 19.6473351 \\
c & 1.08210218 \\
d & 0.0309164915 \\
e & 0.462291896
\end{tabular}

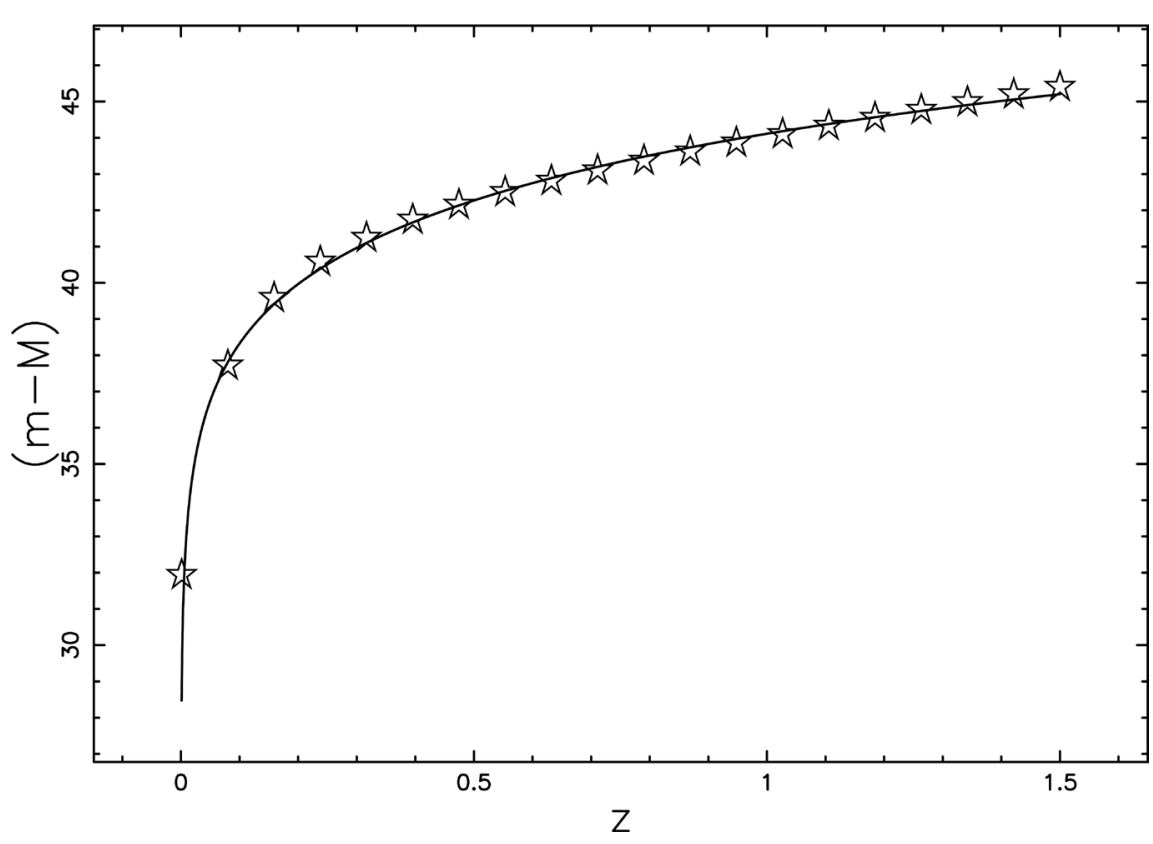

Figure 4. Distance modulus in flat-FLRW cosmology as represented by Equation (22) with parameters as in first line of Table 1 (full line) and minimax rational approximation (empty stars); Union 2.1 compilation.

of SNs of type Ia allows finding the parameters $H_{0}$ and $\Omega_{\mathrm{M}}$ for the two compilations in flat-FLRW cosmology

$$
\begin{aligned}
H_{0}= & (69.77 \pm 0.33) \mathrm{km} \cdot \mathrm{s}^{-1} \cdot \mathrm{Mpc}^{-1}, \quad \Omega_{\mathrm{M}}=0.295 \pm 0.008 \\
& \text { flat-FLRW-Union } 2.1, \\
H_{0}= & (69.65 \pm 0.23) \mathrm{km} \cdot \mathrm{s}^{-1} \cdot \mathrm{Mpc}^{-1}, \Omega_{\mathrm{M}}=0.3 \pm 0.003 \\
& \text { flat-FLRW-JLA, }
\end{aligned}
$$

A first comparison with [8] in the case of the Union 2.1 compilation gives a percentage error $p=0.93 \%$ for the derivation of $H_{0}$ and $p=0.67 \%$ for the derivation of $\Omega_{\mathrm{M}}$. A second comparison can be done with Equation (13) in [22]

$$
H_{0}=(67.27 \pm 0.60) \mathrm{km} \cdot \mathrm{s}^{-1} \cdot \mathrm{Mpc}^{-1}, \quad \Omega_{\mathrm{M}}=0.3166 \pm 0.0084
$$

Planck 2018. 


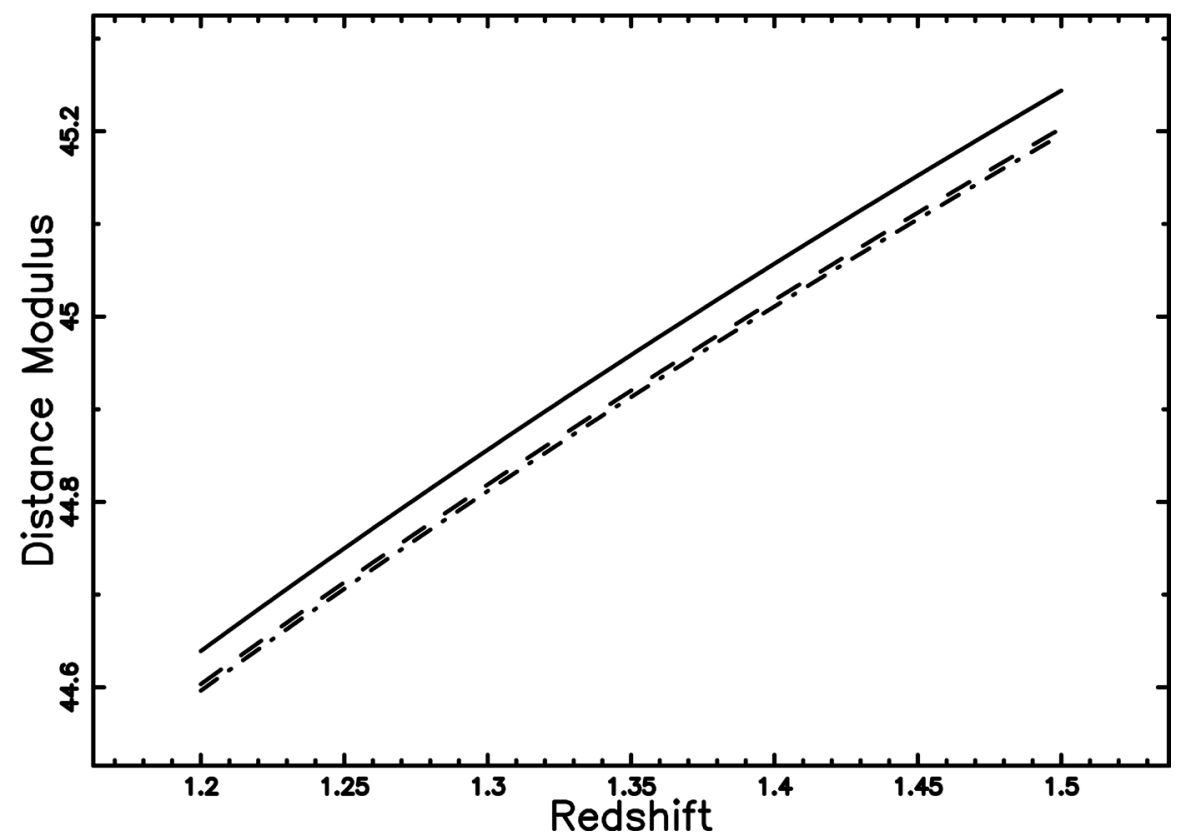

Figure 5. Distance modulus for $\Lambda$ CDM cosmology (full line), flat-FLRW-1 (dot-dash-dotdash line) and flat-FLRW cosmology (dashed line). Parameters as in Table $\mathbf{1}$ and interval of existence $[1.2,1.5]$.

In the case of the Union 2.1 compilation, the percentage error $p=3.71 \%$ for the derivation of $H_{0}$ and $p=6.82 \%$ for $\Omega_{\mathrm{M}}$. A Taylor expansion at low redshift and an asymptotic expansion are presented both for the luminosity distance and the distance modulus. A simple version of the distance modulus is determined through the best minimax rational approximation. Adopting the cosmological parameters found here, the cosmological constant $\Lambda$ turns out to be, for the Union 2.1 compilation,

$$
\Lambda=(1.19457 \pm 0.017) \times 10^{-52} \frac{1}{m^{2}}
$$

flat-FLRW Union 2.1,

or introducing $c=1$ and the Planck time, $t_{p}$,

$$
\Lambda=(3.12046 \pm 0.0462942) \times 10^{-122} \frac{1}{t_{p}^{2}}
$$

\section{flat-FLRW Union 2.1.}

The statistical parameters of the fits are given in Table 1 and Table 2 where the other two models are presented. The values of the $\chi^{2}$ in the above table say that for the Union 2.1 compilation the flat cosmology produces a better fit than the $\Lambda \mathrm{CDM}$ does, but the situation is the reverse for the JLA compilation. As a concluding remark we point out that, thanks to the calibration on the distance modulus of SNs, the differences between the solutions here analyzed are minimum. Therefore a restricted range in redshift should be adopted in order to visualize the diverseness, see Figure 5. 


\section{Conflicts of Interest}

The author declares no conflicts of interest regarding the publication of this paper.

\section{References}

[1] Suzuki, N., Rubin, D., Lidman, C., Aldering, G., Amanullah, R., Barbary, K. and Barrientos, L.F. (2012) The Hubble Space Telescope Cluster Supernova Survey. V. Improving the Dark-Energy Constraints above $\mathrm{Z}$ Greater than 1 and Building an Early-Type-Hosted Supernova Sample. ApJ, 746, 85. https://doi.org/10.1088/0004-637X/746/1/85

[2] Betoule, M., Kessler, R., Guy, J. and Mosher, J. (2014) Improved Cosmological Constraints from a Joint Analysis of the SDSS-II and SNLS Supernova Samples. $A \& A$, 568, A22.

[3] Pen, U.L. (1999) Analytical Fit to the Luminosity Distance for Flat Cosmologies with a Cosmological Constant. ApJS, 120, 49. https://doi.org/10.1086/313167

[4] Adachi, M. and Kasai, M. (2012) An Analytical Approximation of the Luminosity Distance in Flat Cosmologies with a Cosmological Constant. Progress of Theoretical Physics, 127, 145. https://doi.org/10.1143/PTP.127.145

[5] Eisenstein, D.J. (1997) An Analytic Expression for the Growth Function in a Flat Universe with a Cosmological Constant. arXiv:astro-ph/9709054

[6] Liu, D.Z., Ma, C., Zhang, T.J. and Yang, Z. (2011) Numerical Strategies of Computing the Luminosity Distance. MNRAS, 412, 2685.

https://doi.org/10.1111/j.1365-2966.2010.18101.x

[7] Mészáros, A. and Řpa, J. (2013) A Curious Relation between the Flat Cosmological Model and the Elliptic Integral of the First Kind. $A \& A, 556, \mathrm{~A} 13$

https://doi.org/10.1051/0004-6361/201322088

[8] Benitez-Herrera, S., Ishida, E.E.O., Maturi, M., Hillebrandt, W., Bartelmann, M. and Röpke, F. (2013) Cosmological Parameter Estimation from SN Ia Data: A Model-Independent Approach. MNRAS, 436, 854. https://doi.org/10.1093/mnras/stt1620

[9] Press, W.H., Teukolsky, S.A., Vetterling, W.T. and Flannery, B.P. (1992) Numerical Recipes in FORTRAN. The Art of Scientific Computing. Cambridge University Press, Cambridge.

[10] Hogg, D.W. (1999) Distance Measures in Cosmology. arXiv:astro-ph/9905116

[11] Peebles, P.J.E. (1993) Principles of Physical Cosmology. Princeton University Press, Princeton, NJ.

[12] Zaninetti, L. (2016) Pade Approximant and Minimax Rational Approximation in Standard Cosmology. Galaxies, 4, 4. https://doi.org/10.3390/galaxies4010004

[13] Zaninetti, L. (2016) An Analytical Solution in the Complex Plane for the Luminosity Distance in Flat Cosmology. Journal of High Energy Physics, Gravitation and Cosmology, 2, 581. https://doi.org/10.4236/jhepgc.2016.24050

[14] Baes, M., Camps, P. and Van De Putte, D. (2017) Analytical Expressions and Numerical Evaluation of the Luminosity Distance in a Flat Cosmology. MNRAS, 468, 927.

[15] Olver, F.W.J., Lozier, D.W., Boisvert, R.F. and Clark, C.W. (2010) NIST Handbook of Mathematical Functions. Cambridge University Press, Cambridge.

[16] Gupta, R. (2018) SNe Ia Redshift in a Nonadiabatic Universe. Universe, 4, 104. 
[17] Jones, D.O., Scolnic, D.M., Riess, A.G., Rest, A., Kirshner, R.P., Berger, E., Kessler, R., Pan, Y.C., Foley, R.J., Chornock, R., Ortega, C.A., Challis, P.J., Burgett, W.S., Chambers, K.C., Draper, P.W., Flewelling, H., Huber, M.E., Kaiser, N., Kudritzki, R.P., Metcalfe, N., Tonry, J., Wainscoat, R.J., Waters, C., Gall, E.E.E., Kotak, R., McCrum, M., Smartt, S.J. and Smith, K.W. (2018) Measuring Dark Energy Properties with Photometrically Classified Pan-STARRS Supernovae. II. Cosmological Parameters. The Astrophysical Journal, 857, 51.

[18] Ben-Dayan, I., Gasperini, M., Marozzi, G., Nugier, F. and Veneziano, G. (2013) Average and Dispersion of the Luminosity-Redshift Relation in the Concordance Model. Journal of Cosmology and Astroparticle Physics, 6, 2.

[19] Bevington, P.R. and Robinson, D.K. (2003) Data Reduction and Error Analysis for the Physical Sciences. McGraw-Hill, New York.

[20] Remez, E. (1934) Sur la détermination des polynômes d'approximation de degré donnée. Communications of Kharkov Mathematical Society, 10, 41.

[21] Remez, E. (1957) General Computation Methods of Chebyshev Approximation. The Problems with Linear Real Parameters. Publishing House of the Academy of Science of the Ukrainian SSR, Kiev.

[22] Planck Collaboration, Aghanim, N., Akrami, Y., et al. (2018) Planck 2018 Results. VI. Cosmological Parameters. 